\title{
El problema de la productividad en Argentina: perspectivas locales y transnacionales entre el primer peronismo y el frondicismo
}

\author{
O problema da produtividade na Argentina: perspectivas locais e transnacionais \\ entre o primeiro peronismo e frondicismo
}

The problem of productivity in Argentina: local and transnational perspectives between the first Peronism and Frondicismo

Silvia Simonassi*

Universidad Nacional de Rosario (UNR), Rosario, Argentina

\begin{abstract}
RESUMEN: El propósito del artículo es examinar el tema de la productividad del trabajo en Argentina a través de las posturas sostenidas por el Estado y los empresarios, así como las posiciones emanadas de las agencias internacionales - en particular de la Organización Internacional del Trabajo -, para poder determinar tanto las especificidades locales como las convergencias transnacionales. El lapso de tiempo analizado transcurre entre el primer peronismo (1946-1955), el gobierno militar autodenominado Revolución Libertadora (1955-1958) y la gestión del desarrollista Arturo Frondizi (1958-1962). Este último período corresponde al de mayor avanzada en las políticas estatales y empresariales sobre el tema. Se argumenta que la persistencia de perspectivas diversas que convivieron entre funcionarios y empresarios, puede haber permitido a los trabajadores negociar condiciones de trabajo más favorables en el nivel de planta, en un contexto de importantes luchas por evitar la pérdida de las conquistas obtenidas durante el primer peronismo. Esto explica que el denominado "problema de la productividad" reaparecerá en Argentina como una cuestión irresuelta hasta al menos la última dictadura militar argentina de 1976 a 1983.
\end{abstract}

PALABRAS CLAVE: Productividad. Frondicismo. Empresarios. Organización Internacional del Trabajo.

RESUMO: O objetivo do artigo é examinar a questão da produtividade do trabalho na Argentina através da posição assumida pelo Estado e pelos empresários, assim como os posicionamentos emitidos pelas agências internacionais, em particular da Organização Internacional do Trabalho, para poder relacionar as

\footnotetext{
* Profesora titular da Escola de Antropologia e História da Universidad Nacional de Rosario, Rosário, Argentina. Investigadora do Instituto de Investigaciones Socio Históricas Regionales - ISHIR - UNR. Doutora em Humanidades e Artes com menção em Historia. E-mail: silviasimonassi@yahoo.com.ar. http://orcid.org/0000-0002-1496-368X
} 
especificidades locais como as convergências transnacionais. O lapso de tempo analisado transcorre entre o primeiro peronismo (1946-1955), o governo militar de autoimposição A Revolución Libertadora (1955-1958) e a gestão do desenvolvimentista Arturo Frondizi (1958-1962). Este último período corresponde aos maiores avaços nas políticas estaduais e empresariais sobre o tema. Argumenta-se que a persistência de perspectivas diversas que coexistiram entre funcionários e empresários pode ter permitido aos trabalhadores negociar condições de trabalho mais favoráveis no nível da fábrica, num contex to de importantes lutas para evitar a perda das conquistas obtidas durante o primeiro peronismo. Isso explica que o chamado "problema da produtividade" vai voltar na Argentina como uma questão sem solução até a última ditadura militar argentina, de 1976 a 1983.

PALAVRAS-CHAVE: Produtividade. Frondicismo. Empresários. Organização Internacional do Trabalho.

\begin{abstract}
The purpose of this article is to examine the issue of labor productivity in Argentina through the positions supported by the State and the employers, as well as the positions emanating from international agencies, particularly the International Labor Organization, in order to determine the local specificities and transnational convergences. The time period analyzed ranges from the first Peronism (1946-1955), the self-styled military government Revolución Libertadora (1955-1958) and the government of Arturo Frondizi (1958-1962). This last period corresponds to the most advanced state and business policies on the subject. It is argued that the persistence of various perspectives that coexisted between employees and employers, may have allowed workers to negotiate more favorable working conditions at the plant level, in the context of significant struggles to avoid the loss of the conquests obtained during the first Peronism. This explains that the so-called "productivity problem" will reappear in Argentina as an unresolved dispute until the last Argentine military dictatorship from 1976 to 1983
\end{abstract}

KEYWORDS: Productivity. Frondicismo. Capitalists. International Labor Organization.

\title{
El problema de la productividad en Argentina: perspectivas locales y transnacionales entre el primer peronismo y el frondicismo
}

Los debates en torno a la productividad del trabajo a partir del primer peronismo, a las causas que condujeron a su descenso, así como a las soluciones proyectadas y efectivamente implementadas, fueron muy intensos en la historia argentina. Enfrentaron a empresarios, funcionarios, profesionales y trabajadores de forma más o menos institucionalizada en diferentes coyunturas en la segunda mitad del siglo XX.

Sin embargo, su abordaje desde el campo de la investigación histórica ha sido desigual. En parte esto ha respondido a las dificultades de medición de la productividad del trabajo, reconocida por distintos investigadores, no obstante lo cual se han ensayado diversas explicaciones del problema (FERRER, 1956; KATZ, 1967; DORFMAN, 1983). Han sido estudiados los discursos y políticas empresariales y estatales producidos durante el final del peronismo clásico, en particular el Congreso de la Productividad de 1955 (GIMENEZ ZAPIOLA; LEGUIZAMON, 1987; BITRÁN, 1994; SOWTER, 2016). Desde otros ángulos, como la historia obrera y las relaciones obrero-patronales, se ha indagado en la incidencia de estos debates y de las políticas efectivamente aplicadas, en las condiciones de trabajo y en la relación de fuerzas entre las clases (JAMES, 1981; 
KABAT, 2007; SCHIAVI, 2013; SIMONASSI, 1996; 2007). Más recientemente, se ha enfocado el análisis en las políticas estatales sobre la productividad emanadas de la Revolución Libertadora y el frondicismo (JÁUREGUI, 2012; SIMONASSI, 2014a; 2014b).

El propósito del presente artículo es examinar el problema de la productividad del trabajo desde una perspectiva regional, nacional y transnacional. Se estudian las maneras en que el tema fue formulado por diversos actores regionales/nacionales (predominantemente de las áreas industriales de Buenos Aires y Rosario y de la rama metalúrgica) y las posturas emanadas de las agencias internacionales, en particular de la Organización Internacional del Trabajo (OIT), para poder determinar tanto las especificidades locales como las convergencias transnacionales. Para el período bajo estudio el tema - en el marco de las ideas del desarrollo -, formaba parte sustantiva de la agenda de otros organismos internacionales, como la CEPAL (Comisión Económica para América Latina), aunque en este lugar se enfatizan las discusiones en el interior de la OIT por su más directa vinculación con el mundo del trabajo, al representar un terreno de disputa entre empresarios, trabajadores y Estados (CARUSO; STAGNARO, 2017). Los debates allí sostenidos representaban una caja de resonancia de preocupaciones locales e internacionales e influían a la vez, en los debates y políticas sostenidas por los principales actores que allí se expresaban.

El lapso de tiempo abordado transcurre entre el primer peronismo (1946-1955), el gobierno militar autodenominado Revolución Libertadora (1955-1958) y la gestión del desarrollista Arturo Frondizi (1958-1962). Este último período corresponde al de mayor avanzada en las políticas estatales y empresariales, aunque el denominado problema de la productividad reaparecerá en Argentina como una cuestión irresuelta hasta al menos la última dictadura militar argentina de 1976 a $1983 .{ }^{1}$

Se hace foco en la existencia, durante el lapso de tiempo aquí analizado, de significativas diferencias en el campo de las agencias estatales y de las organizaciones empresarias, en torno al carácter técnico o político del problema, abonada por el debate en los estrados internacionales. En este artículo se sostiene que la persistencia de perspectivas diversas que convivieron entre funcionarios y empresarios, representó un factor que permitió a los trabajadores negociar condiciones de trabajo más favorables en el nivel de planta, en un contexto de importantes luchas por evitar la pérdida de las conquistas obtenidas durante el primer peronismo y de las particulares relaciones de fuerza entre las clases.

Para ello recurrimos a fuentes de diversa procedencia: archivos empresariales de Rosario y Buenos Aires, prensa periódica nacional y regional, información de diverso tipo disponible en el Fondo del Centro de Estudios Nacionales (CEN) y en especial el Subfondo Arturo Frondizi de la Biblioteca Nacional, así como material documental de la Organización Internacional del Trabajo. ${ }^{2}$

En una primera parte, presentamos los argumentos que se desplegaron durante el peronismo en el debate en torno a la productividad del trabajo. En un segundo apartado, se muestran los alcances y los límites de los avances sobre la cuestión durante los años posteriores al golpe de Estado de setiembre de 1955, para intentar mostrar que con el triunfo electoral de Arturo Frondizi por la Unión Cívica Radical Intransigente (UCRI), el tema estaba ya instalado tanto a nivel nacional como internacional, con particular referencia a la OIT. En una tercera parte se analizan los discursos y diagnósticos manejados por el gobierno desarrollista, así como las voces que se alzaron y las políticas que emanaron de la OIT, para en la cuarta sección explorar las políticas implementadas a nivel nacional y las lecturas e interpretaciones que suscitaron entre los contemporáneos. Finalmente, se presenta un conjunto de conclusiones. 


\section{Los debates sobre la productividad y la disciplina durante el primer peronismo}

Las apelaciones al incremento de la productividad del trabajo reconocen un momento de condensación del debate durante el primer peronismo. La continuidad de la industrialización sustitutiva tras la Segunda Guerra Mundial, los esfuerzos para lograr el pleno empleo y la distribución de los ingresos a favor de los trabajadores, caracterizaron los primeros años de las políticas económicas del peronismo. Se produjeron también importantes transformaciones en las relaciones de fuerzas en las fábricas, que otorgaron poder a las organizaciones sindicales, las comisiones internas y los cuerpos de delegados. La expansión de las comisiones internas, la introducción de importantes cláusulas en los convenios colectivos que mejoraban las condiciones de trabajo, la imposición de ritmos propios en la ejecución de las tareas, así como importantes logros obreros y sindicales en las pujas distributivas, fueron algunas expresiones de ese proceso, que puso en cuestión la autoridad patronal en los lugares de trabajo.

En esa primera etapa, las referencias al tema entre los empresarios y el gobierno fueron escasas, aunque en Rosario en 1947 se formuló la necesidad de adecuar los aumentos de salarios a la productividad y trabajar en conjunto por "aquietar los ánimos” ante las huelgas. También se propuso acompañar los llamados a la intensificación productiva con premios personales a los técnicos que obtuviesen altos índices de producción. ${ }^{3}$ En Buenos Aires, la Cámara de Industriales Metalúrgicos, daba cuenta de la baja del rendimiento obrero (ausentismo, agitación gremial, huelgas, trabajo a desgano, pérdidas de tiempo, indisciplina), en articulación con el alza de precios de las materias primas y el alto costo de la mano de obra. ${ }^{4} \mathrm{Al}$ año siguiente, el alto índice de ausentismo se anudó en la agenda empresaria a los problemas disciplinarios y el accionar de las comisiones internas y las presiones se profundizaron a partir de 1949 , cuando las condiciones económicas se agravaron. ${ }^{5}$ Las suspensiones y despidos del año 1952, la escasez de divisas y la actuación de las comisiones internas incrementaron las controversias, aunque no fue sino en el año 1954 cuando la patronal metalúrgica se negó a considerar el incremento de salarios desligados del correspondiente aumento de la productividad del trabajo, abriendo una muy significativa coyuntura de huelga (SCHIAVI, 2013).

A partir de entonces, la productividad fue un tema central de la agenda empresaria, tal como lo demostró la organización del Congreso General de la Industria de 1953 y del Congreso de Organización y Relaciones de Trabajo de 1954. Sin embargo, fue el Congreso Nacional de la Productividad y el Bienestar Social, a principios del siguiente año, con participación de la CGE (Confederación General Económica) y la CGT (Confederación General del Trabajo), que convirtió el tema de la productividad en un debate nacional (GIMENEZ ZAPIOLA; LEGUIZAMON, 1988). En el mismo, se enfrentaron las posturas empresarias y sindicales. Las primeras, expresadas a través de José B. Gelbard, máximo líder de la CGE, estuvieron centradas en la necesidad de incrementar los ritmos de producción con la maquinaria disponible y limitar el poder de las comisiones internas, para restituir el "derecho de mando" y evitar que "[...] el delegado toque su silbato en una fábrica y la paralice”. Por otro lado, el farmacéutico Eduardo Vuletich, secretario general de la CGT, sintetizó las posturas obreras, rechazando que los trabajadores debieran someterse a "presiones inhumanas" o a cualquier "aumento incontrolado de la carga de trabajo" y abogó "[...] por una mejor eficiencia del trabajo, es decir, por un menor gasto de esfuerzo físico [...]” a través de la modernización de la maquinaria (JAMES, 1981, p. 324-331). 
El debate se convirtió en una campaña pública de difusión por parte del Estado a través de la Secretaría de Asuntos Técnicos - destinada para las tareas de preparación y divulgación -, que editó un folleto destinado a la difusión de ideas afines al incremento de la productividad. La Comisión organizadora del Congreso de la Productividad abrió un espacio a particulares y entidades para presentar estudios y ponencias que serían recogidas posteriormente. La CGT y la CGE realizaron desde agosto del año anterior sus propias campañas de divulgación, a través de afiches, conferencias y folletos, entre otros medios. Además, diversas publicaciones económicas de la época recogieron opiniones favorables al incremento de la productividad (SOWTER, 2016). La rosarina Federación Gremial, por ejemplo, publicaba en su Boletín la convocatoria de un concurso para el otorgamiento de becas para responder a la pregunta "Qué se entiende por productividad” y "Qué ventajas resultan de una mayor productividad" y convocaba a los asociados a retirar las bases en la sede local de la Federación Económica de la Provincia, perteneciente a la CGE. Difundía además los temas de las diversas ponencias presentadas desde la corporación y sus cámaras. ${ }^{6}$

A pesar de los magros resultados prácticos de esta campaña, interrumpida por el golpe de setiembre de 1955, el problema ya estaba instalado como tal entre los empresarios, centralmente bajo la forma de atar salarios a la productividad, modificar las cláusulas de convenios y limitar la autoridad obrera y sindical en las fábricas. Del gobierno militar dependerían, según su óptica, los avances en este sentido. Para los trabajadores, también quedaba sentada la importancia de los derechos adquiridos y la voluntad de no permitir el retroceso en ese campo.

\section{Problemas estructurales y productividad durante la Revolución Libertadora}

Tras el derrocamiento del gobierno peronista en setiembre de 1955, se pusieron en debate los fundamentos de su política económica, en el controversial marco de una rediscusión de las alianzas sociales y políticas que lo habían sostenido. La idea de "desperonización” sustentada por sus antiguos adversarios, tanto civiles como militares, suponía desmantelar la herencia peronista transformando la matriz económica de la Argentina.

Los problemas de productividad pasaron a formar parte del sombrío balance realizado en torno a la década peronista y en particular, de los informes realizados por la comisión asesora económica conformada por el gobierno militar, donde se destacaba la figura de Raúl Prebisch. El argentino Prebisch era ya por entonces un economista reconocido en el país y en el exterior y desde 1950 se desempeñaba como Secretario Ejecutivo de la Comisión Económica para América Latina (CEPAL), dependiente de las Naciones Unidas, desde donde emanaron las principales ideas que atravesaron el escenario latinoamericano en los cincuenta y encaminaron el debate sobre el desarrollo hacia la industrialización como principal horizonte para los países latinoamericanos. ${ }^{7}$

Sin embargo, los conflictos políticos de la Argentina pos peronista ubicaron a Prebisch en un terreno ligado más bien a políticas económicas de tipo ortodoxo. En efecto, el Informe Preliminar de octubre de 1955, partía de señalar la existencia de severos problemas estructurales provocados por las políticas económicas peronistas, como los de balanza de pagos - generados por el retraso de la producción agropecuaria, la descapitalización de la industria y la ausencia de una política petrolera - y la inflación. Esta última tenía causas complejas desde su perspectiva, como la expansión del crédito bancario y los "masivos aumentos de salarios no acompañados por aumentos de la 
productividad" (PREBISCH, 1956a), que eran resultados de la fortaleza de las comisiones internas y las organizaciones sindicales más que de procesos de modernización o de racionalización de la producción.

Así reaparecería una y otra vez en ese y sus siguientes escritos, el denominado problema de la productividad del trabajo. Como su resolución estaba supeditada a la recuperación del ritmo de desarrollo industrial y a una eventual expulsión de trabajadores, en el contexto en que escribía y pensaba, Prebisch consideraba que el tema debía discutirse cuidadosamente, al representar una fuente de conflictividad. Al analizar el incremento de la proporción de sueldos y salarios en el ingreso total argentino (46,7\% en $1945,59,7 \%$ en 1954 ) afirmaba que el problema era el modo en que ese mejoramiento en los ingresos de obreros y empleados se había producido en Argentina. Un aumento de salarios sin el correspondiente incremento de la productividad hizo necesario "[...] afectar desfavorablemente el ingreso de los productores rurales, con las graves consecuencias que ello trajo consigo, o incurrir en un serio proceso de descapitalización, especialmente en los transportes y la misma agricultura."

Basada en este diagnóstico, la prensa planteaba a fines de 1955 que la situación económica era "delicada" o "grave", apuntando contra la industria excesivamente protegida del anterior gobierno, o augurando "la modificación del concepto desde un estado intervencionista hasta uno que aliente y nutra la iniciativa privada”. ${ }^{8}$ El primer presidente de la dictadura, Lonardi, calificó de “desastrosa” la situación económica argentina.?

Sectores del empresariado y de la prensa se vieron obligados a llamar a apaciguar la "sed de revancha" contra los trabajadores. ${ }^{10}$ Sin embargo, las protestas obreras fueron reprimidas, como el paro general de mediados de noviembre de 1955, que culminó con la declaración de ilegalidad y la intervención de la CGT e inició una etapa de mayor endurecimiento de la represión a los trabajadores, durante el gobierno de Aramburu.

En los documentos dados a conocer a principios del año siguiente (PREBISCH, 1956b), Prebisch insistía en la necesidad de estabilizar la moneda argentina, para lo cual hacía falta la "firme decisión" del gobierno militar. Para ello era necesario incrementar los bienes y servicios en proporción a la programada emisión monetaria, para evitar efectos inflacionarios. Volvía sobre la necesidad de ligar los aumentos de salarios a los correspondientes incrementos en la productividad, llamando a los obreros y empleados a trabajar "mejor”, alentando a producir más, "[...] única forma de mejorar su ingreso y de sacar al país de la encrucijada en que se halla”.

Por su parte, los empresarios debían absorber los aumentos de salarios, formando "juntas gremiales” y suscribiendo convenios de estabilización de precios, recurriendo al Estado solo en los "casos indispensables". Bajo el eufemismo de "abandonar en el trabajo algunas prácticas regresivas", se llamaba a restablecer el sentido de autoridad, jerarquía y responsabilidad. Incremento de la productividad significaba mejor trabajo y no más trabajo, "salvo" cuando se haya trabajado menos de lo que normalmente corresponda, lo cual por cierto configuraba parte del diagnóstico sobre lo sucedido en el ámbito productivo durante el peronismo. Finalmente, el escrito apuntaba hacia la renegociación de convenios colectivos incluyendo fórmulas para atar los salarios al incremento de productividad. ${ }^{11}$

Ese fue el marco en que se avanzó en la legislación, en particular con el decreto 2739/56 por medio del cual se levantaron los condicionamientos legales que limitaban los incrementos de productividad, indicando los parámetros a los cuales debían ajustarse los convenios colectivos. 
Quedaron autorizados los desplazamientos de mano de obra para reorganizar los procesos de trabajo, se permitió la implantación de incentivos "morales y materiales", se permitió a las empresas concertar con sus trabajadores acuerdos especiales sobre sistemas de incremento de productividad y se anularon todas las cláusulas de convenio que atentaran contra los aumentos de productividad. Se definía un cambio notable en el sistema de "promociones", atadas a criterios como "idoneidad y contracción al trabajo”, además de "antigüedad, disciplina y asistencia al trabajo”. Finalmente se creó un Tribunal Arbitral con representación de las tres partes, que actuaría en cuestiones de estabilidad, remuneración, categoría, jornada legal, salud y seguridad en el trabajo. ${ }^{12}$

Fundados en esta normativa, los industriales metalúrgicos del país se negaron a mediados de 1956 a discutir condiciones de trabajo y plantearon tratar solamente los salarios y las cláusulas del convenio prorrogado que implicasen trabas a la productividad..$^{13}$ La emblemática huelga metalúrgica de 1956 se extendió por casi dos meses y su resultado fue un aumento muy inferior al inicialmente solicitado por la UOM (Unión Obrera Metalúrgica) aunque las cláusulas de convenio referidas a la productividad no se modificaron. ${ }^{14}$ Las medidas represivas y los despidos continuaron en diversas fábricas, en un contexto de conflictividad en sectores como la construcción, calzado, gráficos, textil, frigorífico y construcción naval, donde las huelgas fueron también declaradas ilegales y los huelguistas perseguidos (JAMES, 1990; SCHNEIDER, 2005). No obstante, se ha puesto en duda la efectividad y extensión de los cambios, que fueron "[...] menos definidos de lo que la patronal hubiese deseado", en tanto la resistencia obrera nacida en especial de las comisiones internas y del nuevo activismo surgido en estos años, frenaron su aplicación (JAMES, 1990). En efecto, en la segunda mitad de 1957, los empresarios rosarinos consideraban que los aumentos de salarios por productividad continuaban representando una "discusión pendiente". 15

Los industriales metalúrgicos habían comenzado a abordar el tema desde múltiples, heterogéneas y más complejas perspectivas, en un Congreso Nacional reunido en 1956. Recomendaron mejoras en lo que consideraban "factores directos" que incidían en la productividad: el ambiente de trabajo, los equipos y maquinarias, los materiales, la provisión de energía y combustibles y el "factor humano". En este sentido, insistieron en un mayor acercamiento entre las organizaciones patronales y obreras, la creación de comisiones o institutos sociales para problemas asistenciales; "[...] fomentar el interés del personal de los establecimientos en el desarrollo y progreso del mismo, estimulando el aporte de ideas para el perfeccionamiento de los métodos de producción o de los productos mismos mediante premios, retribuciones y otros sistemas, así como informándole de las líneas generales de su desenvolvimiento”. Planteaban además propiciar la inmigración de trabajadores especializados, promover a los trabajadores por pruebas de suficiencia ante jurados competentes, la fijación de salarios incentivados y ajustados según zonas y denunciaron “[...] el sistema de los pagos retroactivos como atentatorio contra la estabilidad económica y social del país”, exigiendo su abolición.

También demandaban al Estado por los “factores concurrentes” como el crédito bancario y las tarifas de transportes. El reequipamiento de las industrias dependía, según su óptica, de la cuestión impositiva, el cambio preferencial, los permisos automáticos de importaciones y el crédito. Propusieron también la creación de un organismo empresario para "[...] procurar el aumento de la productividad y la racionalización de la producción industrial [...]”(CONGRESO NACIONAL DE LA INDUSTRIA METALÚRGICA, 1956, p. 85-94), financiado por sus organizaciones. Postularon también la adecuación de los contenidos de la enseñanza técnica, la colaboración para 
el mejoramiento de métodos, la incorporación de sistemas de costos industriales, la reorganización del sistema de estadísticas y censos para adaptarlo a las necesidades de la industria. Finalmente, plantearon la organización de un Instituto Tecnológico Nacional que estudiase nuevas fuentes de materias primas, buscar aplicaciones a las materias primas nacionales, desarrollar nuevos métodos industriales para su mejor aprovechamiento y fijar controles de calidad para las exportaciones de manufacturas. ${ }^{16}$

En esta problematización de la cuestión de la productividad del trabajo se encuentran ecos de debates más generales, de carácter internacional, iniciados ya a principios de la década en organismos como la Organización Internacional del Trabajo (OIT). En rigor, el tema preocupaba a otros organismos de carácter internacional, como la CEPAL, tal como quedaba claro en el Manifiesto Prebisch, el documento de 1949 donde se sentaban las bases del pensamiento estructuralista y el impulso a la industrialización y la modernización agrícola de los países latinoamericanos (PREBISCH, 1949). En la OIT, por su parte, había asumido en 1949 como Director General el estadounidense David Morse, quien en el primer informe a la Conferencia Internacional ubicó a la cuestión de la productividad en el centro de la escena. ${ }^{17}$

De modo que el debate comienza a aparecer de modo recurrente a partir de entonces en el discurso del Director General, entre los asistentes a las Conferencias y profesionales convocados. Así, una Conferencia de expertos se reunió en Ginebra en diciembre de 1952, exponiendo “[...] la necesidad de establecer una colaboración entre la dirección y los trabajadores” (OIT, 1953, p. 110-111). En esa oportunidad se concluyó que era necesario contar con "un movimiento sindical libre y poderoso y la práctica de la negociación colectiva” y que los gobiernos establecieran mecanismos de consulta a empleadores y trabajadores. Esa reunión reconoció la diferencia de criterios, recomendando a la OIT “[...] fomentar una comprensión más amplia del significado de la mayor productividad [...]” (OIT, 1953, p. 110-111) y de sus resultados; establecer acuerdos sobre cómo lograr que dicho incremento signifique un incremento del bienestar económico y social de la comunidad y brindar asistencia y asesoramiento técnicos a tal efecto. Además de esta reunión, otras específicas de ramas industriales mostraban la creciente preocupación mundial sobre el tema. ${ }^{18}$

El aumento de la productividad en todos los sectores era, para el Director General, el único camino hacia el desarrollo, aunque no garantizaba por si solo el progreso social. De allí que el enfoque otorgado integrara aspectos educativos, sociales y técnicos y promoviera una mejora del bienestar socioeconómico general. Así, "[...] las buenas relaciones laborales y las políticas satisfactorias de salarios y empleo [constituían] partes integrales de los programas para incrementar la productividad [...]” con el trasfondo de la guerra fría y el peligro de agitación comunista ante el descontento social (MAUL, 2019, p. 173-174).

En la Conferencia de 1953, se consideró medular el problema de la productividad, entendido como la oferta insuficiente de bienes y servicios y la ineficaz utilización de los recursos existentes. La amplitud de la mirada en torno a la productividad queda en evidencia en las cuestiones que la afectaban: "formación profesional, colocación, salarios, seguridad e higiene, seguridad social, condiciones de trabajo", todas cuestiones que atravesaban las preocupaciones del organismo internacional. ${ }^{19}$

Sin embargo, los desacuerdos en torno a la importancia de elevar la productividad y las formas de lograrlo, fueron expuestos por el Director General y se hicieron visibles a lo largo del debate. Las dudas en torno a la mecanización y los riesgos de desempleo, las diferencias surgidas ante las 
propuestas de participación de los trabajadores en las ganancias obtenidas a partir del incremento de la productividad, las diferencias entre los diferentes sectores de la economía, fueron centrales tanto en el informe del Director General como en la Conferencia de 1955. Allí, el representante de los empresarios argentinos reconoció la importancia de los informes elaborados por la OIT y de la reunión de expertos de 1952 en las posturas sustentadas durante el Congreso de la Productividad y el Bienestar Social, así como la asistencia de dos enviados de la Organización. Por su parte, la representación obrera, en la voz de Vuletich, expresó una opinión crítica hacia la Organización y sobre la incidencia práctica de las resoluciones. En la misma dirección que las posturas defendidas en el orden local, dejó sentada una perspectiva acerca del incremento de la productividad que demandaba un consenso previo en términos de beneficio común, legislación adecuada, una poderosa organización sindical y “[...] la garantía de que los beneficios no serán exclusivos de los poseedores del capital”. Por su parte, el Informe del Director General transparentaba un debate que por entonces estaba centralmente anclado en los países desarrollados. ${ }^{20}$

De modo que, durante estos años, en el orden internacional se había instalado el tema, aunque recién a partir de entonces los países en vías de desarrollo entraban en la agenda de preocupaciones, al compás de los avances en los procesos de industrialización. A nivel nacional, luego del golpe de estado de 1955 y en el marco de una manifiesta reformulación de las relaciones de fuerza entre las clases, se pusieron en marcha una serie de dispositivos para introducir modificaciones en las relaciones de trabajo tendientes a restaurar la potestad patronal en planta y a suprimir cláusulas de convenios que obstaculizaban los incrementos de productividad. No obstante, estos cambios fueron mucho menos efectivos de lo esperado por el empresariado y abrieron la posibilidad de que al menos ciertos sectores del empresariado industrial discutieran en términos más amplios, menos anclados en las "herencias" del peronismo, los problemas de productividad en las fábricas - como quedó expresado en el Congreso Metalúrgico de 1956 -, aunque sin abandonar como objetivos inmediatos atar los incrementos de salarios a la productividad y limitar el poder obrero en las plantas, en un contexto de persistente y firme resistencia obrera.

\section{Ideas y recomendaciones para incrementar la productividad durante el frondicismo}

Las políticas industriales implementadas durante el gobierno de Arturo Frondizi (1958-1962) se correspondieron con una nueva etapa de la industrialización en Argentina, derivada del agotamiento de los capitales nativos y orientada al otorgamiento de generosas concesiones al capital extranjero para invertir en áreas estratégicas del desarrollo industrial. De allí que durante estos años se abordó el problema de la productividad desde una perspectiva más general y técnica y se persiguió como condición para lograrlo, el objetivo de disciplinamiento de la fuerza de trabajo.

Cuando Frondizi se hizo cargo del gobierno, debido a su pacto electoral con el peronismo, revocó los decretos anti sindicales y aumentó en un $60 \%$ los salarios congelados desde febrero de 1956. No obstante, a partir de principios de 1959 los cambios en la política económica contenidos en el Plan de Estabilización, incrementaron la ya persistente resistencia obrera. A medida que la racionalización del sector público avanzaba, las contiendas obreras se endurecieron, aunque la represión, en particular con la aplicación del plan CONINTES, hizo retroceder cuantitativamente la cantidad de huelgas. ${ }^{21}$ 
Durante estos años se llevó adelante un verdadero "movimiento por la productividad", una "campaña” orientada a reformular la industria nacional dentro de parámetros de eficiencia productiva y disciplina industrial (JÁUREGUI, 2012). Se diseñaron organismos destinados a abordar el problema, se encargaron investigaciones, se contrataron expertos y se avanzó en modificaciones en las cláusulas de los convenios colectivos de trabajo. Las ideas sobre el desarrollo atravesaron instituciones, agencias estatales y el mundo académico y configuraron el marco a partir del cual se trazaron las políticas económicas.

Para Frondizi, los aumentos de productividad debían resultar del esfuerzo de empresarios, trabajadores y gobierno. El gobierno debía proteger y fomentar la industria a través de un plan de largo alcance. Los empresarios capitalizando sus empresas, mejorando equipos e instalaciones, racionalizando sus métodos de producción y creando condiciones favorables entre sus operarios. Los trabajadores debían trabajar mejor, capacitarse técnicamente y utilizar racionalmente la jornada de trabajo (JÁUREGUI, 2012). Tras anunciar el incremento de salarios de "carácter excepcional” del 60\%, Frondizi dejaba "[...] perfectamente sentado que un aumento efectivo de salarios solo podrá resultar de un aumento concreto de los bienes producidos por el conjunto de la Nación” (FRONDIZI, 1978, p. 75).

Que el problema adquirió centralidad durante su gobierno quedó asentado en varios materiales producidos en particular por la Secretaría de Relaciones Económico Sociales, como el que afirmaba, a fines de 1959, que "la naturaleza de la crisis económica argentina" estaba asociada a una "crisis de productividad”, de "orígenes más profundos” que las fallas del material humano o la defectuosa utilización del material mecánico. ${ }^{22}$ Señalaba la insuficiente incorporación de mano de obra en los sectores más productivos y aún la escasa productividad allí registrada. Tras un diagnóstico de la situación argentina y las inversiones por sectores, el informe recomendaba una serie de posibles soluciones: mejorar la capacidad de importación, aumentar las inversiones de capital extranjero en sectores dinámicos de la economía, mejor utilización de los equipos existentes (a pesar de que en paralelo se reconoce su obsolescencia), reorientación de las inversiones hacia el sector de bienes de capital y desarrollo de la siderurgia. ${ }^{23}$

Otro informe señalaba que era posible lograr importantes incrementos en la rentabilidad de las empresas y en el producto bruto nacional "[...] sin adicionales inversiones de capital y sin desplazamiento de personal que pueda producir perturbaciones sociales” (CHAPIRO, 1958, s.p.). Los empresarios debían tomar decisiones con fundamentos científicos, como invertir en tecnología y garantizar su uso a pleno, realizar previsiones de materias primas, programar la producción, realizar tareas de mantenimiento preventivas, de las instalaciones térmicas y de mínimos stocks. Para el gobierno estas "recomendaciones técnicas" resultaban unilaterales, pues carecían de una mirada de las razones profundas de la industrialización en la Argentina, que debía reorientarse hacia sectores básicos. Pero dejaba abierta la posibilidad de estudiar el tema a través de un Consejo de Promoción Industrial, o la realización de jornadas e instancias de articulación universitaria y empresarial en un Instituto de Investigaciones de la Industria. ${ }^{24}$

En rigor, la preocupación por el incremento de la productividad y la racionalización desbordaba las fronteras nacionales y regionales y continuaba propiciando intercambios transnacionales. La Oficina Internacional del Trabajo (OIT) en la 42을 Conferencia realizada en 1958 adoptó diversas resoluciones sobre el tema: una de ellas indicaba la necesidad de otorgar prioridad al tema de la capacitación de la mano de obra para fomentar programas de desarrollo económico, adelantando 
lo que las Naciones Unidas denominaron a partir de principios de la década del sesenta Decenio para el Desarrollo. Otra de las resoluciones, enfatizó la necesidad de incorporar métodos racionales de dirección con el objetivo de fomentar el desarrollo económico y aumentar la productividad, para lo cual era incumbencia de la OIT instar a los gobiernos - en el camino de las misiones de productividad - a implementar diversos mecanismos de investigación, de capacitación y de difusión para lograr ese fin (como escuelas superiores de administración de empresas). La gestión eficaz de las empresas constituía una condición indispensable para "asegurar que los resultados favorables de la formación profesional y de los esfuerzos de los trabajadores se traduzcan plenamente [...] en una mayor producción, y [...] que se vean compensados por la elevación del nivel de vida y el mejoramiento de las condiciones de trabajo" (OIT, 1958, p. 3-9). En esa oportunidad recomendaron también, intensificar las investigaciones, el intercambio de informaciones, la asistencia técnica y la conformación de institutos y centros para la formación y el estudio de las relaciones entre empleadores y trabajadores. ${ }^{25}$

La OIT cristalizaba los esfuerzos por incrementar la productividad a través de las misiones de asistencia técnica, una de las cuales fue enviada a fines de 1957 a Argentina y transformada en misión de largo plazo, con el criterio de que la eficacia y los efectos duraderos podían garantizarse a través de la asistencia de varios años y distintos profesionales. En 1959 se agregaron dos expertos y se acordó prestar colaboración con integrantes de Naciones Unidas. En 1958 el Fondo Especial de Naciones Unidas había permitido a la OIT obtener financiamiento de otras fuentes. En 1960 se creó el Instituto Internacional de Estudios Laborales (IIEL), dedicado a la investigación y la formación entre otras cuestiones vinculadas a las relaciones laborales. ${ }^{26}$ Algunas iniciativas se vieron truncadas, como en el año 1960, cuando se suprimió una reunión sobre problemas de aumento de productividad para América Latina.

Sin embargo, la visión que el delegado gubernamental por Argentina mostró en la 43o Conferencia reunida a mediados de junio en Ginebra evaluaba que en el accionar de la OIT existían escasos elementos para países como Argentina, que "[...] no reviste las características típicas de los países subdesarrollados; sin embargo, tampoco ha alcanzado el nivel industrial de algunas de las naciones aquí representadas. Esta etapa de evolución social y económica suscita problemas para cuya solución pacífica no se cuenta siempre con métodos adecuados.” (OIT, 1960, p. 390). Para el argentino, varios convenios adoptados "[...] no dan orientación para problemas como los que mi país sufre, sea por haber sido propiciados teniendo en cuenta las estructuras jurídicas y las tradiciones de los países altamente industrializados, o bien por atender solamente a las necesidades de aquellos que acaban de comenzar a asomarse a la revolución industrial” (OIT, 1960, p. 390). ${ }^{27}$

Si bien demandaban asistencia técnica y formación profesional a migrantes como parte de la necesidad más general de formación de personal especializado, el delegado se detuvo en cuestiones sindicales, al exhibir "[...] el riesgo de que las partes recurran a medidas de fuerza que no sólo perjudican el normal desarrollo de la vida económica, sino que obligan a adoptar medidas destinadas a proteger al resto de la comunidad. Mi país ha sufrido y sufre agudamente las consecuencias de estos problemas [...]" (Ibidem, p. 391), hacia los cuales las normas internacionales exhiben "ambigüedades". Y continuaba:

Aunque el derecho de huelga está constitucionalmente garantizado en Argentina, ello no implica que las autoridades no deban preocuparse por evitar con los medios legales a su alcance los movimientos de esta índole, con su secuela de graves pérdidas en la producción. 
Los sistemas tradicionales de conciliación obligatoria y arbitraje voluntario no son suficientes para detenerlos cuando tienen motivación política o son provocados por luchas sindicales internas. (Ibidem, p. 391)

Las críticas realizadas no impidieron que Argentina se postulara como sede para la realización de la Conferencia Regional de Estados Americanos, aunque muestra la heterogeneidad de situaciones que convivían en las Conferencias y la centralidad que la conflictividad obrera, en el marco de un sistema consolidado de convenciones colectivas, revestía a los ojos de los representantes del gobierno argentino. ${ }^{28}$

Algo similar mostraban las quejas de los empresarios argentinos, que llegaron a la OIT en la 450 Conferencia de Ginebra de 1961, cuando demandaron sindicatos que "[...] no sean instrumentos de movimientos políticos, generalmente contrarios al fondo de los ideales de libertad de la clase trabajadora [...]" (OIT, 1961, p. 29) y dejaron sentado que "[...] la huelga solo es admisible como último recurso... pero es inaceptable en los diferendos jurídicos [...]” (OIT, 1961, p. 29). Más claramente, repudiaron "[...] enérgicamente las huelgas políticas y las formas arteras de la acción gremial directa, como es el llamado 'trabajo a reglamento' o la disminución deliberada del ritmo de trabajo.” (OIT, 1961, p. 106-107) ${ }^{29}$ El Director General, por su parte, colocó en su informe a Argentina como un ejemplo destacado de los países en vías de desarrollo que más días perdidos por huelga ostentaba en términos comparativos, configurando más de la mitad del total de jornadas perdidas por un conjunto de once países que contabilizaba el organismo en esa categoría. ${ }^{30} \mathrm{La}$ airada respuesta del metalúrgico Augusto T. Vandor, representante de los trabajadores a la Conferencia, estuvo centrada en reseñar las legítimas y genuinas causas de la elevada conflictividad en Argentina, así como la ofensiva represiva desatada desde el Estado. Allí, el entonces Secretario General de la UOM y representante de las "62 Organizaciones” peronistas, se pronunció a favor de un plan de desarrollo económico con asistencia y colaboración, que no significara "regresión en materia social y la negación o mutilación de nuestras libertades a la vez que la castración económica del país”. Vandor mencionó críticamente también, el escaso análisis dedicado a América Latina en el Informe del Director General. ${ }^{31}$

\section{Las políticas estatales de incremento de la productividad y sus repercusiones}

En el marco de los crecientes debates en torno a la industrialización como condición para el desarrollo, se implementaron diversas políticas estatales. En 1957 había sido finalmente creado en Argentina el Instituto Nacional de la Productividad, como organismo descentralizado dependiente del Ministerio de Comercio e Industria y con participación empresaria a través de la Unión Industrial Argentina (UIA) y obrera, a través de la CGT. ${ }^{32}$

A fines de 1959 el Fondo Especial de las Naciones Unidas aprobó la creación del Instituto de la Productividad propuesto por el gobierno argentino y sobre esa base, sumando a la Asociación Argentina de Productividad y los "[...] centros de productividad que se crearán bajo sus auspicios [...]”, se acordó sobre fines del año siguiente ampliar los servicios para disponer cada vez mayor cantidad de personal de dirección competente, especialistas en técnicas de mejoramiento de la productividad, jefes de servicios y personal calificado para satisfacer las necesidades de la industria. El proyecto estaría a cargo de la OIT y la Secretaría de Estado de Industria y Minería, tendría una duración de cinco años y un presupuesto de casi dos millones de dólares, los cuales casi en partes iguales compartían el Fondo Especial y el Gobierno Argentino. ${ }^{33}$ 
En el marco de esos acuerdos se dispuso la llegada al país de cincuenta y ocho expertos, la participación de ciento dieciseis técnicos argentinos y el otorgamiento de veinticuatro becas para realizar estudios de perfeccionamiento en el exterior. ${ }^{34}$ Se creó además un Centro de Productividad entre el INTI (Instituto Nacional de Tecnología Industrial) y la Asociación Argentina de la Productividad, para difundir este tema, formar y capacitar recursos en las empresas y realizar proyectos técnicos e investigaciones especiales. ${ }^{35}$ Contemplaba la formación de expertos en técnicas de producción, control de calidad, de mantenimiento, capacitación de supervisores y enseñanza técnica en coordinación con el recién creado CONET (Consejo Nacional de Enseñanza Técnica). El proyecto recibió el apoyo de la UIA por cuanto contemplaba el trabajo conjunto con entidades privadas. ${ }^{36}$

La UIA fue también anfitriona del Ingeniero David Moushine, jefe de la misión de expertos de la OIT que participaban del plan de incremento de la productividad, para explicar a los asociados sobre la importancia del tema en la "recuperación económica del país" ${ }^{37}$ Según su óptica, los expertos eran “[...] el botón de arranque del automóvil. El automóvil tendrá que moverse con su propio motor" (LA RAZÓN, 29/1/1961, p. 5), para sintetizar la política de asesoramiento articulada con la concesión de becas a técnicos argentinos en el exterior. ${ }^{38}$

Un año después de creado el Centro de Productividad había recibido expertos extranjeros, había fortalecido relaciones con instituciones educativas, organizaciones empresarias, técnicas y obreras. Editaba y distribuía un Boletín Mensual denominado Productividad, dictaba conferencias y cursos, como el de Introducción al Estudio del Trabajo con presencia de empresarios y dirigentes sindicales. Se habían creado los Centros de Productividad del Transporte de Buenos Aires, en la Cámara Argentina de la Construcción y en la empresa Siam. Según el informe, habían llegado cuatro técnicos extranjeros y se programaba la llegada de otros diez para el año 1962. ${ }^{39}$

El "movimiento por la productividad” alcanzó diversos ámbitos, como la prensa periódica, la radio y otros medios de difusión. Por ejemplo, los diarios La Razón, La Prensa y Democracia, entre otros, publicaban notas sobre el tema, ${ }^{40}$ se realizaron actividades como una mesa redonda con la exposición de distintos profesionales ${ }^{41} \mathrm{y}$ audiciones radiales especiales en el marco del ciclo "Meridiano del Trabajo", difundido por el Ministerio de Trabajo y Seguridad Social. ${ }^{42}$

Para algunos especialistas, la productividad consistía en lograr mayor cantidad, mejor calidad y menor costo, pero consideraban que esto debía lograrse a través del aumento del rendimiento y nunca del esfuerzo de los trabajadores "[...] del desgaste que puede sobrepasar límites científicamente establecidos”. Según esta postura, a la sociedad le interesaba más “[...] el concepto de ciclo o período vital productivo del hombre, es decir, la cantidad de años de vida durante los cuales es capaz de producir más de lo que gasta”. La fatiga “[...] reversible, no acumulativa, no altera la salud. Por el contrario, mantiene al organismo en mejores condiciones lo mismo que la gimnasia o el deporte racionalmente practicado" (LA RAZÓN, 20/12/1960, s.p). ${ }^{43}$

Otro experto señalaba que la mayor productividad se traducía en "[...] más larga vida, pues al pretenderse el aprovechamiento más racional de la mano de obra con fines fundamentalmente económicos... toma en especial consideración el factor humano como elemento productor al que hay que cuidar con más atención que a la máquina, pues en definitiva rinde más que ella” (LA RAZÓN, 19/2/1961, s.p.). ${ }^{44}$

En el tema de salarios, se insistía en atarlos a la productividad, en avanzar sobre determinadas cláusulas de convenios y sobre la "indisciplina sindical", traducida en frecuentes paros "por cualquier motivo”. La idea de flexibilizar remuneraciones surgía de la crítica a las escalas salariales 
unificadas a nivel nacional, obtenidas a través de los convenios colectivos de trabajo. Finalmente, la crítica a "la demagogia peronista", los “[...] demagogos de los partidos burgueses [...] y principalmente los activistas del comunismo internacional” cerraba la opinión sobre el poder de presión de los trabajadores y sus organizaciones. ${ }^{45}$

Diferente y cambiante fue la postura asumida desde la Revista Qué, desde donde se refirió inicialmente al "mito de la baja productividad obrera", mostrando a través de la relación entre el volumen físico de la producción y las horas-obrero trabajadas, el incremento producido a pesar de la escasa renovación de maquinarias. Afirmaban "[...] el problema de la productividad en la industria no reside tanto en postular una incentivación del trabajo obrero, como en renovar equipos y maquinarias anticuadas con las que se ven obligadas a trabajar hoy la mayoría de las fábricas, por la carencia de divisas necesarias para adquirirlas". ${ }^{46}$ El equipamiento de la industria resultaba fundamental, para lo cual consideraban prioritario desarrollar la industria de máquinas herramientas, junto al autoabastecimiento energético y el desarrollo de la siderurgia. ${ }^{47}$

Sin embargo, poco después en la misma publicación se afirmaba que era preciso hacer un uso más racional de la mano de obra disponible, tanto en el sector industrial como en la administración pública, sin acudir a los despidos en masa. Para ello proponía la creación de nuevas fuentes de trabajo en los sectores promocionados (petróleo y siderurgia), la actividad minera para sustituir importaciones, pero también en nuevos sectores como el alimenticio, promoviendo hábitos alimentarios alternativos al alto consumo de carne. La situación en las fábricas era descripta ahora de manera distinta: los trabajadores estaban sometidos a “un desgaste de energías no muy grande”, en fábricas con baja productividad y alto número de trabajadores con salarios insuficientes, para resolver lo cual deben acudir a un segundo empleo, por lo cual proponían "reordenar todo el sistema de trabajo". ${ }^{48}$

Más aún, el salario por rendimiento pasó a ser promovido desde las páginas de la publicación, cuestionando el mecanismo de negociaciones colectivas que profundizaban la brecha de salarios entre trabajadores. Se trataba de "[...] tomar de un sector para dárselo a otro" (Subrayado en el original). Si bien reconocía que este debate remitía al menos a 1952 y se había profundizado durante la "Revolución Libertadora”, se detenían en describir los disímiles objetivos y el contexto político sindical. Ahora era primordial enfatizar en la creación de riqueza como presupuesto para el incremento de salarios, al tiempo que, según los redactores, se había revertido la política antisindical de los años precedentes. Quedaba sin embargo, culminar el proceso de normalización gremial.49

La revista defendía así los lineamientos del gobierno nacional en relación a la política económica y sindical, en las diversas coyunturas. En efecto, a principios de 1959, el Plan de Estabilización, traducido en reducción del empleo estatal, congelamiento de salarios del personal y subas de tarifas e impuestos, estaba en marcha.

Las anteriores opiniones, de conjunto, confirman que más allá del discurso tecnocrático propio de los funcionarios desarrollistas y de los expertos en productividad, se insistía en realizar los ya señalados esfuerzos por avanzar sobre las conquistas obreras en los lugares de trabajo, en una Argentina fuertemente marcada por la herencia peronista.

Sin embargo, en las principales áreas industriales argentinas, la paradigmática y prolongada huelga metalúrgica de 1959 y su resolución, exhibió la ambigüedad de la resolución del problema. Si bien los artículos 82 y 83 del convenio colectivo de trabajo firmado en 1960 reglamentó las comisiones internas y recortó los derechos de los delegados y el sindicato, su efectiva implementación nuevamente dependió de las relaciones de fuerza tramadas en los lugares de trabajo. ${ }^{50}$ 


\section{Conclusiones}

Los límites que comenzaba a evidenciar el proceso de industrialización, los problemas de obsolescencia técnica y las transformaciones que durante el peronismo se produjeron en la relación de fuerzas entre las clases a nivel de planta, produjeron el primer gran debate en torno a aquello que se consideraba una de las debilidades del proceso de industrialización argentino: la baja productividad obrera. En esos debates de finales del peronismo - sobre 1954 y 1955 -, se expusieron los argumentos centrales que dividieron aguas entre trabajadores y empresarios. Los primeros responsabilizaban de esa situación a los empresarios, por su escasa disposición a invertir en tecnología mientras los segundos argumentaban que las comisiones internas, los delegados sindicales, las cláusulas de los convenios, las huelgas y el ausentismo eran responsables del decrecimiento de los índices de producción en relación a los obreros ocupados. Desde entonces la lucha empresaria y estatal se concentró en limitar los derechos adquiridos por los trabajadores durante el peronismo a nivel de planta. Para ellos, el problema de la productividad se entrelazó fuertemente con el imperativo de disciplinamiento social y laboral.

De allí que, tras la caída del peronismo, los empresarios vieron la posibilidad de superar rápidamente esa situación de "prepotencia” obrera en las fábricas. De hecho, entre 1955 y 1958 el gobierno logró avanzar en modificar la legislación para introducir cambios en los convenios colectivos, pero su aplicación dependió notablemente de la fuerza que las distintas organizaciones y las bases sindicales ostentaron para frenar los avances sobre conquistas históricas.

Pero la "Libertadora" fue también el momento en el cual se hicieron oír voces dentro del mundo empresario, profesional y de los elencos gubernamentales, que subrayaron la existencia de problemas estructurales que excedían la variable del comportamiento y la organización obrera y había que comenzar a resolver. El marco general de ese debate eran las ideas vinculadas al estructuralismo cepalino y al desarrollo. Prueba de ello fueron las heterogéneas conclusiones que sobre el tema produjo el Congreso de la Industria Metalúrgica y la creación del Instituto Nacional de la Productividad.

Fue un momento en que retumbaron los ecos de los debates internacionales, iniciados a principios de la década e incrementados a medida que la parte del mundo subdesarrollada o en desarrollo - potenciada por el proceso de descolonización - adquiría preponderancia, donde el incremento de la productividad aparecía asociado a la formación profesional, el empleo, los salarios, la higiene, la seguridad social y las condiciones de trabajo.

En Argentina, durante los años frondicistas se redoblaron los esfuerzos estatales y la decisión empresaria de abordar el tema, aunque desde diferentes ópticas y perspectivas. La profundización del proceso de industrialización mediante el aliento a la industria pesada de capitales extranjeros fue el marco en el cual se comenzó a operar para mejorar los índices de productividad. Dando forma a una agresiva campaña por el incremento de la productividad, con la llegada de expertos extranjeros, con la capacitación de profesionales argentinos en el exterior, mediante programas radiales y campañas de prensa, creando Centros de Productividad y encargando informes técnicos, el gobierno de Frondizi otorgó un tratamiento más global al problema de la productividad del trabajo. La actividad de la OIT, las misiones técnicas, la creación del Fondo Especial de Naciones Unidas que permitió incrementar las partidas de dinero, la centralidad otorgada al debate manifestada en reuniones específicas, no solo fueron el marco, sino que permitieron el despliegue de las políticas estatales argentinas. 
Por otro lado, durante estos años se avanzó en el país en las modificaciones de las cláusulas de convenios, como aconteció con el convenio metalúrgico de 1959. Y tras el triunfo de la revolución cubana y su posterior radicalización, se produjo un notable recrudecimiento de la represión, cristalizado en la aplicación del plan CONINTES y en los avances en la racionalización del estado. Por eso el frondicismo ha sido caracterizado como el momento de mayor embestida - tras los primeros intentos empresarios y gubernamentales de 1954 y la propia "Libertadora" - sobre las conquistas obreras que ponían límites a la explotación patronal y su autoridad en las plantas. Sin embargo, la real aplicación de las cláusulas de convenio dependió de las relaciones de fuerza establecidas en las plantas, que remitía a las conquistas logradas durante las décadas precedentes y el poder de los sindicatos y su representación en las fábricas.

La multiplicidad de perspectivas que atravesó el universo de expertos, funcionarios y empresarios en torno a las mejores maneras de encarar los aumentos de la productividad del trabajo, explica los escasos y desiguales resultados de las políticas implementadas, así como la persistencia del problema más allá del año 1962. Ello potenció la capacidad de los trabajadores de luchar por conquistas consideradas históricas e irrenunciables. El "Decenio para el Desarrollo" anunciado por las Naciones Unidas abrió nuevos desafíos para los trabajadores del sector industrial en el marco de las transformaciones estructurales que se desplegaron en la economía argentina.

\section{Referencias}

ALTIMIR, Oscar et al. Los instrumentos de promoción industrial en la postguerra. Desarrollo Económico, n. 21, p. 89-144, 1966.

BITRÁN, Rafael. El Congreso de la Productividad. La reconversión económica durante el segundo gobierno peronista. Buenos Aires: El bloque editorial, 1994.

BRENNAN, James; ROUGIER, Marcelo. Perón y la burguesía argentina. El proyecto de un capitalismo nacional y sus límites (1946-1976). Buenos Aires: Lenguaje Claro Editora, 2013.

CARUSO, Laura; STAGNARO, Andrés. Por una historia regional de la OIT. In: CARUSO, Laura; STAGNARO, Andrés (coords.). Una historia regional de la OIT. Aportes sobre regulación y legislación del trabajo latinoamericano. La Plata: FAHCE-UNLP, 2017. p. 13-21.

DICÓSIMO, Daniel. Más allá de la fábrica. Los trabajadores metalúrgicos. Tandil, 1955-1962. Buenos Aires: La Colmena, 2000.

DORFMAN, Adolfo. Cincuenta años de industrialización en la Argentina, 1930-1980. Desarrollo y perspectivas. Buenos Aires: Ediciones Solar, 1983.

FERRARO, Nícolas; SCHIAVI, Marcos. La resistencia obrera en el largo plazo: racionalización industrial y luchas obreras en una coyuntura crítica (1954-1956). El caso metalúrgico. Hindustri@, año 6, n. 11, p. 37-59, segundo semestre 2012.

FERRER, Aldo. El estado y el desarrollo económico. Buenos Aires: Raigal, 1956.

FERRERAS, Norberto O. La misión de Stephen Lawford Childs de 1934: la relación entre la OIT y el Cono Sur. In: HERRERA LEON, Fabián; HERRERA GONZALEZ, Patricio. América Latina y la Organización Internacional del Trabajo. Redes, cooperación técnica e institucionalidad social, 1919-1950. México: UMSNH, UM, UFF, 2013. p. 161-195.

FRONDIZI, A. Precios, salarios y situación económica. Mensaje radiofónico transmitido el 13 de mayo de 1958. Mensajes Presidenciales - 1958-1962, Tomo I. Buenos Aires: Centro de Estudios Nacionales, 1978.

GIMENEZZAPIOLA, M.; LEGUIZAMÓN, C. M. La concertación peronista de 1955: el Congreso de la Productividad. In: TORRE, J. C. (comp.). La formación del sindicalismo peronista. Buenos Aires: Legasa, 1987. p. 321-358. 
JAMES, Daniel. Racionalización y respuesta de la clase obrera: contexto y limitaciones de la actividad gremial en la Argentina. Desarrollo económico, n. 83, p. 321-349, 1981.

JAMES, Daniel. Resistencia e integración. El peronismo y la clase trabajadora argentina, 1946-1976. Buenos Aires: Sudamericana, 1990.

JÁUREGUI, Aníbal. La productividad del trabajo: otra “batalla” del desarrollismo (1955-1962). Anuario CEEED, n. 4, p. 191-228, 2012.

KABAT, Marina. El Congreso de la Productividad de 1955, un análisis desde los enfrentamientos en la industria del calzado. Razón y Revolución. n. 17, p. 90-103, 2007.

KATZ, Jorge. Características estructurales del crecimiento industrial argentino - 1946-1961. Desarrollo Económico, n. 26, p. 59-76, 1967.

MAUL, Daniel. La Organización Internacional del Trabajo. 100 años de políticas sociales a escala mundial. Ginebra: OIT, 2019.

PLATA, Véronique. La difusión de las normas internacionales del trabajo en Venezuela, 1936-1939. Una práctica de cooperación técnica internacional en la OIT. In: HERRERA LEON, Fabián; HERRERA GONZALEZ, Patricio. América Latina y la Organización Internacional del Trabajo. Redes, cooperación técnica e institucionalidad social, 1919-1950. México: UMSNH, UM, UFF, 2013. p. 127-160.

PONTORIERO, Esteban. Estado de excepción y contrainsurgencia: el Plan CONINTES y la militarización de la seguridad interna en la Argentina (1958-1962). Contenciosa, n. 4, p. 1-16, 2015.

PREBISCH, Raúl. El desarrollo económico de la América Latina y algunos de sus principales problemas. Santiago de Chile: CEPAL, 1949.

PREBISCH, Raúl. Informe preliminar acerca de la situación económica de Argentina. El trimestre económico, n. 23, p.1-41, enero-marzo 1956a.

PREBISCH, Raúl. Moneda sana o inflación incontenible - Plan de restablecimiento económico. Buenos Aires: Presidencia de la Nación, 1956b.

PREBISCH, Raúl. Iniciativa privada, la industria y la programación del desarrollo económico. Discurso pronunciado en la audición ‘Argentina Económica’ por la Radio del Estado, 10 de octubre de 1956c.

ROUGIER, Marcelo; ODISIO, Juan. "Argentina será industrial o no cumplirá sus destinos”. Las ideas sobre el desarrollo nacional (1914-1980). Buenos Aires: Imago Mundi, 2017.

SCHIAVI, Marcos. Algunas consideraciones sobre poder obrero y productividad en el primer peronismo: la mirada de los industriales metalúrgicos (1946-1955). In: DICÓSIMO, Daniel; SIMONASSI, Silvia. Trabajadores y empresarios en la Argentina del siglo XX. Rosario: Prohistoria Ediciones, 2011. p. 175-190.

SCHIAVI, Marcos. El poder sindical en la Argentina peronista, 1946-1955. Buenos Aires: Imago Mundi, 2013.

SCHNEIDER, Alejandro. Los compañeros, Trabajadores, izquierda y peronismo, 1955-1973. Buenos Aires: Imago Mundi, 2005.

SCODELLER, Gabriela. Educar en derechos laborales: políticas y acciones desplegadas por la OIT en América Latina durante los años 1950-1970. In: CARUSO, Laura; STAGNARO, Andrés (coords.). Una historia regional de la OIT. Aportes sobre regulación y legislación del trabajo latinoamericano. La Plata: FAHCE-UNLP, p. 213254, 2017.

SIMONASSI, Silvia. Productividad y disciplina en las fábricas metalúrgicas del Gran Rosario: una mirada desde el periódico de la Asociación de Industriales Metalúrgicos de Rosario (1974-1981). Papeles de Trabajo CESOR, n. 3, p.1-15, 1996

SIMONASSI, Silvia. “A trabajar y muzzarella”. Prácticas y políticas de disciplinamiento laboral en la industria metalúrgica de Rosario, 1974-1983. Historia Regional, n. 25, p. 57-82, set. 2007.

SIMONASSI, Silvia. Las ideas económicas del gobierno de la "Revolución Libertadora” y los industriales rosarinos (1955-1958), ponencia presentada al IV Congreso Latinoamericano de Historia Económica (CLADHE IV), Bogotá, Colombia, julio 2014a.

SIMONASSI, Silvia. Repensando el problema de la productividad en la Argentina de los años frondicistas, ponencia presentada a las XXIV Jornadas Argentinas de Historia Económica, oct. 2014b. 
SIMONASSI, Silvia. EI Plan de Lucha de la CGT argentina y las dinámicas regionales de la conflictividad en el Gran Rosario hacia mediados de la década de 1960. Revista Trabajo y Sociedad, n. 33, p. 391-411, 2019.

SIMONASSI, Silvia; VOGELMANN, V. “Pegar un chiflido y juntar gente”. La conflictividad obrera en el Gran Rosario, 1955-1962. In: SIMONASSI, Silvia; DICÓSIMO, Daniel (coords.). Trabajadores y sindicatos en Latinoamérica. Conceptos, problemas y escalas de análisis. Buenos Aires: Imago Mundi, 2018. p. $151-171$.

SOWTER, Leandro. La experiencia del Congreso de la Productividad y la política de la cooperación económica durante el peronismo. Temas y Debates, n. 32, p. 135-154, julio/diciembre 2016.

STAGNARO, Andrés; CARUSO, Laura. “El mundo obrero latinoamericano ante la Organización Internacional del Trabajo a lo largo del siglo XX”. Anuario del Instituto de Historia Argentina, v. 17, n. 1, e 032, p.1-5, jun. 2017.

\section{Notas}

${ }^{1}$ Si bien esta hipótesis no es abordada en este artículo, ha sido explorada en Simonassi (2007). Alli planteamos la necesidad de identificar las diferencias entre incremento de la intensidad y de la productividad del trabajo. La primera supone aumento de gasto humano de energía mediante el uso de la tecnología existente, para obtener mayor cantidad de productos-mercancías. En el segundo caso, se trata de mayor producción con idéntico ritmo y cantidad de trabajadores, es decir, supone mayor "eficacia técnica". Consultar también James (1981), Schiavi (2011; 2013). Históricamente, la delimitación de los alcances y los mecanismos de incremento de la "productividad" del trabajo ha sido un territorio de disputa entre empresarios, trabajadores y Estado. El incremento de la productividad aparece como un objetivo del proceso de racionalización, aunque no el único, al constituir este último “[...] un proceso integral en la organización de la producción asociado a la voluntad de adaptación del capital a una nueva fase de acumulación" (FERRARO; SCHIAVI, 2012, p. 39).

2 Disponible en: https://labordoc.ilo.org/discovery/search?vid=41ILO_INST:41ILO_V2\&lang=en.

${ }^{3}$ Editorial. Los siete peligros. Revista de la Federación Gremial del Comercio y la Industria de Rosario, en adelante. Revista, n. 237, jun. 1947. El propio Perón aludió al tema del rendimiento y la productividad del trabajo en un ciclo de audiciones radiales (SOWTER, 2016).

${ }^{4}$ Metalurgia, n. 94, marzo 1948. Que los problemas principales desde la óptica patronal eran el ausentismo, la indisciplina, las conquistas en torno a la salubridad en el trabajo y la extendida legislación laboral, lo recordaba el vicepresidente primero de CAIM, Alberto Schärer. El descenso de la producción en la industria metalúrgica. Metalurgia, n. 100, setiembre 1948.

${ }^{5}$ Resolvemos el problema del ausentismo obrero. Revista, n. 250, julio 1948. El nuevo servicio de Policía del Trabajo. In: Boletín Informativo Semanal de la Federación Gremial del Comercio y la Industria de Rosario en adelante. Boletín, n. 78, 10 de julio de 1948; Ecos de la comida del día de la industria, Revista, n. 256, enero 1949. Consultar: Schiavi (2011; 2013) y Kabat (2007). Explicaciones sobre la centralidad que adquirió el problema de la productividad en el marco de los cambios en la política económica del período 1949-1952 en el país. Brennan y Rougier (2013); Sowter (2016).

${ }^{6}$ Boletín, n. 403, 12 mar. 1955

${ }^{7}$ Existe una abundante bibliografía sobre el papel desempeñado por Prebisch en el país, desde su actuación como Subsecretario de Hacienda, integrante del equipo de Pinedo y gerente del Banco Central de la República Argentina en los años treinta en adelante, incluyendo su participación en el Plan Pinedo y su actuación como docente en la Facultad de Ciencias Económicas de la Universidad de Buenos Aires en la década del cuarenta. Igualmente importante es la producción que destaca su proyección internacional desde esos mismos años, fundamentalmente tras asumir como Secretario Ejecutivo de la CEPAL en 1950, hasta su alejamiento en 1963. La CEPAL se convirtió en una de las principales usinas de elaboración de ideas sobre el desarrollo y en la búsqueda de instrumentos y mecanismos para lograr esa meta en las economías latinoamericanas. Consultar, entre otros: Rougier y Odisio (2017).

${ }^{8}$ El Litoral, Santa Fe, 8, 21, 25 de octubre de 1955, reproduciendo una declaración de la Cámara Argentina de Comercio.

${ }^{9}$ El Litoral, 27 de octubre de 1955

${ }^{10}$ El Litoral, 30 de octubre y 15 de noviembre de 1955, Boletín, n. 439, 26 de noviembre de 1955 y Cámara de Industriales Metalúrgicos de Rosario, Actas del Consejo Directivo (CIM-CD), 371, 17/11/1955, fo. 233-236.

${ }^{11}$ Prebisch recomendó también la incorporación de Argentina al Fondo Monetario Internacional. Al tiempo que formulaba metas de corto plazo, Prebisch adelantaba la necesidad de elaborar un plan de industrialización a largo plazo, mediante el concurso de capitales extranjeros, con énfasis en las industrias estratégicas, como se expresa entre otras intervenciones en: PREBISCH,1956b. Para un análisis de las múltiples repercusiones del Informe, tanto entre economistas e intelectuales como empresarios, consultar, entre otros Rougier y Odisio (2017, p. 208-222) y Simonassi (2014).

${ }^{12}$ Boletín, n. 458, 14/4/1956, Metalurgia, n. 183, julio de 1956. 
${ }^{13}$ Metalurgia, n. 183, julio de 1956.

${ }^{14}$ Metalurgia, n. 186, octubre-noviembre-diciembre de 1956; Ferraro y Schiavi (2012).

${ }^{15}$ Federación Gremial del Comercio y la Industria de Rosario, Actas de Consejo Directivo (FG-CD), t. 7, n. 533, 7/8/1957, p. 59 y ss.

${ }^{16}$ Tema IV, Productividad y racionalización de la producción. In: Congreso Nacional de la Industria metalúrgica, 16, 17 y 18 de noviembre de 1956. Rosario, 1956. p. 85-94.

${ }^{17}$ Tal como ha analizado Daniel Maul, Morse asumió con cuarenta años de edad, con una relevante carrera dedicada a la cuestión social y laboral, tanto de alcance nacional como internacional. Cumplió un papel fundamental en la ampliación del Plan Marshall a los países subdesarrollados anunciada por Truman en los Cuatro Puntos de su discurso de asunción. Justamente fue en el Punto Cuatro donde se prometía "proporcionar recursos tecnológicos y conocimientos especializados a las regiones subdesarrolladas del mundo para ayudarles a aumentar su productividad" (MAUL, 2019, p. 162), lo cual estableció un vínculo estrecho entre la OIT en su nueva etapa y el Plan Marshall. Las Naciones Unidas y en particular la OIT, constituirían para Truman un vehículo ideal para canalizar la ayuda técnica. Para Morse, la asistencia técnica “[...] podía convertir a la OIT en un instrumento más eficaz para el bloque occidental y para situar las actividades de la Organización en la primera línea del conflicto con la Unión Soviética" (MAUL, 2019, p. 164). En 1950, la OIT junto con otros organismos internacionales de las Naciones Unidas conformaron el "Programa Ampliado de Asistencia Técnica de las Naciones Unidas", contribuyendo, según la lectura de Maul, a disipar al menos la desconfianza sobre la influencia de los EEUU en la organización, aunque sin que eliminarla del todo (STAGNARO; CARUSO, 2017). A partir de entonces se abre un conjunto de transformaciones en el interior de la OIT que caracterizan el período durante el cual Morse actúa como Director General: en 1954 es readmitida la URSS y la presencia de los países "en desarrollo" creció exponencialmente, estimulada por el proceso de descolonización. De 1948 a 1970, los países miembros pasaron de 55 a 121, lo cual la obligó a readaptar sus políticas y programas. Así, la década del cincuenta ubicó al problema de los derechos humanos y cuestiones como la libertad sindical, el trabajo forzoso y la discriminación en un nuevo marco, ahora caracterizado por las relaciones entre el Occidente y el Sur Global (MAUL, 2019).

${ }_{18}$ Organización Internacional del Trabajo (OIT), Memoria del Director General de la Oficina Internacional del Trabajo, Informe I de la Conferencia Internacional del Trabajo. Los problemas del trabajo en el mundo. Productividad y bienestar. La situación económica y social. Actividades de la OIT. (1953, p. 110-111). Las conclusiones del encuentro están contenidas en International Labour Organization, Report of the Meeting of Experts on Productivity in Manufacturing Industries, Geneva, December 1952.

${ }^{19}$ OIT. Memoria del Director General... 1953, cit. p. 3.

20 OIT. Actas de la Trigésima octava Conferencia Internacional de Trabajo, Ginebra, 1955, p. 216-311; Memoria del Director General a la Trigésima Octava reunión, Ginebra, 1955.

${ }^{21}$ Durante 1959 grandes huelgas fueron reprimidas duramente, como la acontecida en el Frigorífico Lisandro de la Torre y la nacional bancaria. Al año siguiente se aplicó formalmente el Plan CONINTES, mediante el cual ante las "graves perturbaciones" que se sucedían, el gobierno autorizaba a las Fuerzas Armadas a "proceder con toda rapidez y absoluta energía" para restablecer el "orden público", subordinando a las policías provinciales. Al respecto, se ha resaltado que el Plan estuvo destinado a colocar bajo la esfera de las FFAA el combate contra el enemigo interno, asociado a los altos niveles de conflictividad social y política que afrontó el gobierno frondicista (PONTORIERO, 2015). Sobre la resistencia obrera del período en diferentes regiones y ramas, incluso contra las políticas de racionalización en el sector estatal, hay una cada vez más importante producción. Entre otros, (DICÓSIMO, 2000; SCHNEIDER, 2005; SIMONASSI; VOGELMANN, 2018).

${ }^{22}$ Presidencia de la Nación Argentina. Secretaría de Relaciones económico-sociales: Memorandum sobre la productividad en Argentina, El problema de la productividad en la Argentina y Memorandum Elementos para una exposición sobre "productividad y organización empresaria", 18 de noviembre de 1959, Fondo Centro de Estudios Nacionales (CEN), Archivos y Colecciones Particulares, Biblioteca Nacional de la República Argentina (BNRA). Entre los insumos para elaborar el informe se encuentra transcripto un artículo de la Revista Internacional del Trabajo de abril de 1953 con conceptos de Jean Fourastie sobre la productividad en los países occidentales. Este material se halla reunido en una carpeta titulada "El problema de la productividad en la Argentina".

${ }^{23}$ Ibidem.

${ }^{24}$ CHAPIRO, Jorge, Las técnicas modernas en la dirección de empresas, 1958. El Ing. Chapiro era presidente de Chapiro y Asociados Consultores Industriales, Archivo CEN-BNRA.

25 OIT. Resoluciones adoptadas en la 42 reunión de la Conferencia Internacional del Trabajo, Ginebra, 4-26 de junio de 1958, p. 3-9.

${ }^{26}$ OIT. Memoria del Director General a la Conferencia Internacional del Trabajo de 1959 (parte II), Decimocuarto Informe de la Organización Internacional del Trabajo a las Naciones Unidas. Actividades de la OIT, Informe I (Parte II), Ginebra, Oficina 
Internacional del Trabajo, 1960. Para un análisis de las misiones de asistencia técnica en un período previo, consultar: Plata (2013); Ferreras (2013).

${ }^{27}$ OIT. Actas de la cuadragésima cuarta Conferencia Internacional del Trabajo, Oficina Internacional del Trabajo, Ginebra, 1960, p. 390. La intervención reflejaba la efectiva preocupación de la OIT por los países recientemente incorporados por el proceso de descolonización.

${ }^{28}$ Ibidem, p. 391. Es de destacar que a la Conferencia de 1960 no asistió la delegación obrera ni empresaria. La cuestión sindical exhibía claras diferencias en los diversos países representados en las Conferencias, de allí las "ambigüedades" señaladas.

${ }^{29}$ OIT. Actas de la Cuadragésima quinta reunión de la Conferencia Internacional del Trabajo, Ginebra, 1961, p. 29.

${ }^{30}$ OIT. Memoria del director general, Informe I, Parte I. Relaciones laborales. Problemas actuales y perspectivas para el porvenir, Ginebra, 1961, p. 106-107.

${ }^{31}$ OIT. Actas de la Cuadragésima quinta reunión de la Conferencia Internacional del Trabajo, Ginebra, 1961, p. 309-311. La atención prestada por el organismo a América Latina durante la década de 1960 se encuentra analizada en Scodeller (2017) y Stagnaro y Caruso (2017, p. 3) han subrayado la pérdida de relevancia relativa de América Latina en su relación con la OIT, desde mediados de la década de 1950 hasta fines de la siguiente, como consecuencia de la descentralización propuesta des de la propia organización y por la atención prestada a los países recientemente incorporados como resultado del proceso de descolonización. Sin embargo, reconocen la existencia de vínculos consolidados que permitieron mantener una agenda propia, entre las cuales incorporan a las misiones.

32 Boletín, n. 535, 26/10/1957.

${ }^{33}$ Presidencia de la Nación Argentina. Ministerio de Relaciones Exteriores y Culto, Memorandum para el Presidente: Plan de Operaciones para la creación del Instituto de la Productividad, 11 octubre de 1960, Archivo CEN/BNRA.

34 “Se pone en marcha un plan de productividad financiado por la UN”. La Razón, 5/3/1960. Los artículos de prensa citados a partir de este momento (diarios La Razón, La Prensa, Democracia, El Correo de la Tarde y Crítica), pertenecen al archivo CEN. Están contenidos en la carpeta "Productividad" y dan cuenta de la preocupación persistente y sostenida del gobierno sobre el tema.

35 “Quedó integrado ayer un Centro de Productividad”. La Prensa, 3/9/1960.

36 “La importancia de la Productividad señaló un experto de la OIT”. La Razón, 25/11/1960. El Centro estaba dirigido por los presidentes y directores del Instituto Nacional de la Productividad, y la Asociación Argentina de la Productividad y llevaría a la práctica "el proyecto de adiestramiento y productividad industrial presentado por las autoridades argentinas y aprobado por el Fondo Especial de las Naciones Unidas el 9 de diciembre de 1959": "Quedó integrado ayer un Centro de Productividad". La Prensa, 3/9/1960. Por su parte, el INTI realizaba investigaciones de procesos industriales, estudios sobre normas y estandarización, pruebas de resistencia de materiales y estudios de control de calidad de productos: Altimir et al. (1966).

37 “La importancia de la Productividad señaló un experto de la OIT”. In: La Razón, 25/11/1960, p. 6. De Moushine la prensa informaba que se desempeñaba en Tel Aviv en el cargo de director del Instituto Israelí de Productividad, habiendo recibido en 1957 una beca de la OIT para el estudio y comparación de los centros de productividad de Europa Occidental: “Se pone en marcha un plan de productividad financiado por la UN". La Razón, 5/3/1960.

38 “De productividad en la Argentina habló el representante de la OIT”. La Razón, 29/1/1961, p. 5.

39 “Elevaron al Presidente de la Nación el informe de un año de labor del Centro de la Productividad”. Democracia, $17 / 10 / 1961$, p. 5.

40 La Razón. 12/11/1959; $1^{\circ}$ al 6/2/1960. Durante esta semana se publicó una serie de notas titulada "Los secretos de la productividad". Democracia, 3/5/1961,15/5/1961, 13/10/1961.

41 “Hay que inclinar la balanza”. La Razón, 20/12/1960, p. 14.

42 “Programa de incremento de la productividad en nuestro país”, Crítica, 22/1/1961, p. 7; “De productividad en la Argentina habló el representante de la OIT". La Razón, 29/1/1961, p. 5 y “Trabajador, consumidor y empresario deben compartir la productividad, expresó un técnico”, La Razón, 19/2/1961, p. 7.

43 “Hay que inclinar la balanza”, La Razón, 20/12/1960.

44 “Trabajador, consumidor y empresario deben compartir la productividad, expresó un técnico”, La Razón, 19/2/1961.

${ }^{45}$ San Martín, Salvador. El problema laboral y la productividad. Correo de la Tarde, 5/4/1961, p. 23 y 11/4/1961, p. 28. Posturas similares se sostenían en: “Los secretos de la productividad. Fórmula número 1”, La Razón, 3/2/1960, donde además se cuestionaba la fijación de tareas y el principio "un hombre una máquina”. 
46 “El mito de la baja productividad obrera”. Qué sucedió en 7 días, n. 185, 10 de junio de 1958, p. 12-13.

47 “Equipar, mecanizar, pero... ¿cómo?, Qué sucedió en 7 días, n. 188, 1 de julio de 1958.

48 “Más productividad sí, pero con más fuentes de trabajo”, Qué sucedió en 7 días, n. 222, 24 de febrero de 1959.

49 “Salario por rendimiento: debate necesario en los plenarios”, Qué sucedió en 7 días, n. 227, 31 de marzo de 1959, p. 9. Subrayado en el original.

${ }^{50} \mathrm{CIM}$, Convención colectiva 55/60 para empleados y obreros de la industria metalúrgica, Rosario, 1960. Existen diversas interpretaciones en torno de los avances de la legislación y las relaciones de fuerza entre las clases luego de 1960 en diferentes regiones: James (1990), Dicósimo (2000), Schneider (2005), Simonassi (2019).

Submetido em: 20/03/2020

Aprovado em: 24/06/2020 